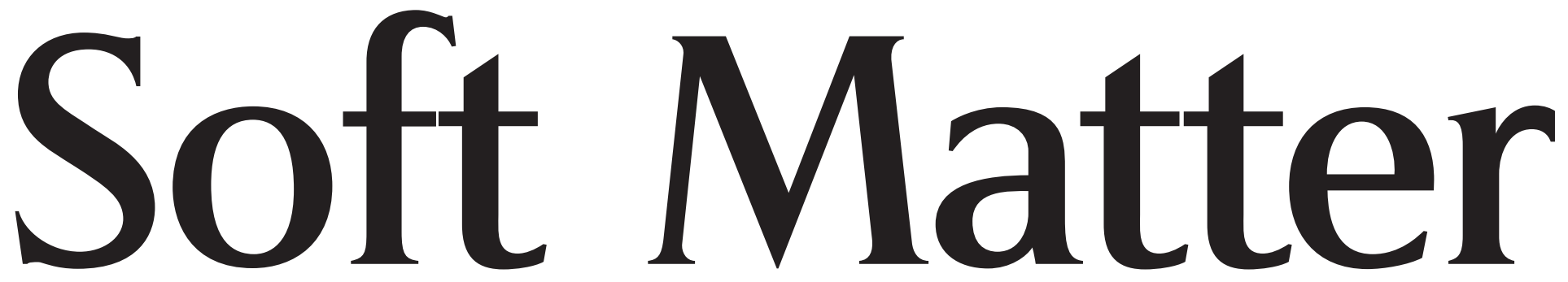

www.rsc.org/softmatter

Volume 7 | Number 5 | 7 March 2011 | Pages 1545-2164

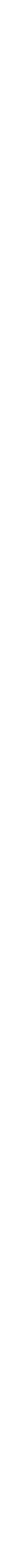




\title{
Liquid crystalline behavior of a coarse-grained model of shape-persistent macrocycles with flexible attractive chains
}

\author{
Carlos Avendaño and Erich A. Müller* \\ Received 1st September 2010, Accepted 11th November 2010 \\ DOI: $10.1039 / c 0 s m 00905 a$
}

Shape-Persistent Macrocycles (SPMs) are synthetic organic macromolecules characterized by a rigid open hoop or ring with flexible side groups pointing either towards the inside or outside of the structure. In a previous work, we have studied the liquid crystalline behavior of a Coarse-Grained model of SPMs using Molecular Dynamics simulations [C. Avendaño and E. A. Müller, Phys. Rev. E, 2009, 80, 061702]. In the aforementioned model, the system tends to organize in a rather unusual smectic-A phase in which the particles are aligned perpendicularly to the layers, i.e. the system presents antinematic order. In this work, this model is extended to study the effect of flexible chains attached to the macrocycles on the self-assembly of SPMs. Considering flexible chains linked to the SPM backbone allows a qualitative comparison with real systems where the chains play an important role in the supramolecular organization of the system. In our model particles are composed of a rigid hexagonal arrangement of 24 soft-repulsive spheres with chains of attractive spheres grafted into the cycle. Two different cases (isomers) have been considered in this work depending whether the attached chains are pointing inward (intraannular substituents) or outward (extraannular substituents) from the rigid structure. The isomers self-assemble into contrasting liquid crystalline phases upon temperature quenching from an isotropic state. It is observed how systems with extraannular substituents tend to order at high densities into a lamellar phase with the chains laying in between the layers. The orientation of the rings in the layers shows antinematic order. For SPMs with intraannular substituents, the systems exhibit a transition from an isotropic phase to an hexagonal-disordered columnar phase, where the attractive chains fill the inner voids of the ring and help to stabilize the columns. The liquid crystalline phases in both models are influenced by the amphiphilic nature of the different components of the model.

\section{Introduction}

More than three decades have passed since Chandrasekhar described liquid crystalline (LC) molecules based on discotic structures of molecules having a polyaromatic core with flexible side groups. ${ }^{1}$ Since then, many other examples of discotic liquid crystals materials have been reported in the literature (see for example the reviews by Bushby and Lozman ${ }^{2}$ and by $\mathrm{Kumar}^{3}$ ). Apparently, the first theoretical explanation of formation of LC phases in these systems was given by Onsager showing that, by purely entropic arguments, anisotropic disc-like particles can exhibit an Isotropic-Nematic transition. ${ }^{4,5}$ Computer simulation experiments of discotic hard bodies have confirmed that it is also possible to form stable columnar $(\mathrm{Col})$ structures by the stacking of discotic particles. ${ }^{6-8}$ On larger length scales, colloidal platelet suspensions confirm this behavior. ${ }^{9-11}$ The advantage of the colloidal particles over their molecular counterpart is that their size allows their direct observation in real space, ${ }^{12}$ and may be used to test theoretical predictions.

In the last decade interest has arisen for non-conventional discotic liquid crystals. In this context non-conventional should be understood as a variation of the typical "core-like" molecule,

Department of Chemical Engineering, Imperial College London, South Kensington Campus, London, SW7 2AZ, UK. E-mail: e.muller@, imperial.ac.uk either by decoration or by fundamental changes in the design. Shape-persistent macrocycles (SPMs) lie into this category. SPMs are $\pi$-conjugated organic ring-like macromolecules with a rigid structure typically comprised of meta and para aromatic groups connected directly by acetylene or diacetylene groups in such a way that the final structure cannot collapse; $c f$. Fig. 1 (a)(b). Interest in the supramolecular arrangement of functionalized SPMs has increased considerably in view of their potential application as a building block for more complex structures and their ability to form interesting 2D patterns at interfaces. ${ }^{13-23}$

Different kind of mesophases have been observed in SPMs depending on several factors such as size of the macrocycle cavity, properties of the functional groups belonging to the rigid macrocycle, structure and characteristics of the side chains attached to the backbone, among others. Apparently, the first SPM showing interesting LC behaviour was reported by Höger et al. ${ }^{15}$ This system is comprised of a rigid macrocycle, made of 10 phenylene subgroups, connected through acetylene units with alkyl chains attached to the backbone. Two primary architectures can be envisioned depending on whether the phenylene group, to which the alkyl chain is directly attached, can or cannot freely rotate in the ring structure. Surprisingly, only when the phenylene group is able to rotate, does the system exhibit LC phase behavior -in this case a nematic $(\mathrm{N})$ phase, which is observed in the temperature range between $185-207{ }^{\circ} \mathrm{C}$. This feature is possible only when the lateral flexible chain can fold 


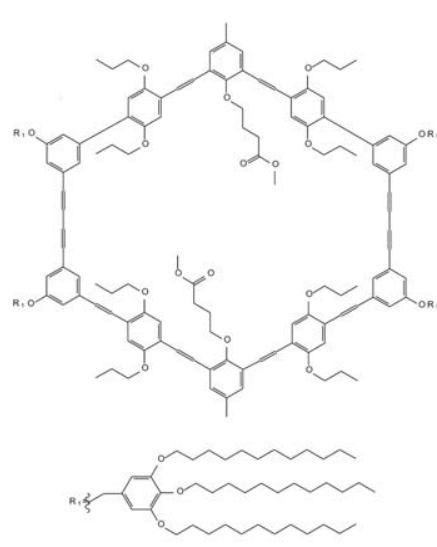

(a)

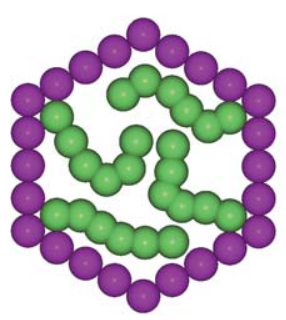

(c)

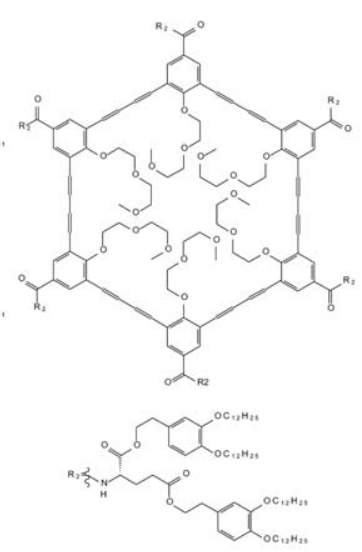

(b)

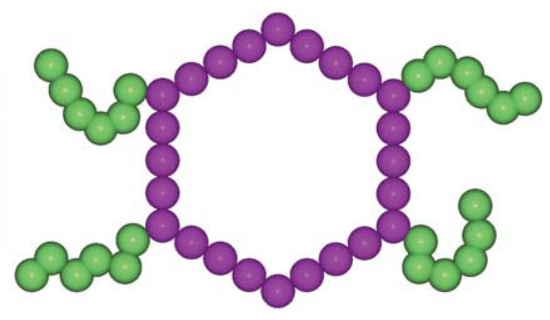

(d)
Fig. 1 (a)-(b) Chemical structures of the amphiphilic SPMs reported by Fischer et $a l .{ }^{24}$ and Shimura et al. ${ }^{23}$ respectively; (c) and (d) are representations of the CG models of amphiphilic SPMs used in this work. (c) SPM model with intraannular flexible chains attached to the rigid backbone; (d) SPM model with extraannular flexible chains. In both models, the principal molecular axis, $\hat{\mathbf{u}}$, is pointing out in a direction perpendicular to the plane of the ring (toward the reader). In both CG models, dark spheres (purple) represent the soft-repulsive beads of the core and light spheres (green) are the attractive chains.

back into the cavity of its own ring. In this system, the discotic geometry is preserved by the rigid periphery, but the core is not always void as it may be filled by its own alkyl chains, i.e. it is a liquid crystal system with an inverted structure. The system with the extraannular alkyl chains does not present stable thermotropic mesophase presumably due to the frustration between the molecular anisotropy and the empty space of the ring which is filled by alkyl chains of neighbor rings. ${ }^{15,24,25}$

Efforts to functionalize these molecules in order to design stable Col phases (tubular superstructures), instead of only $\mathrm{N}$ phases, have also been reported. Fischer et al., for example, reported a SPM system with intraannular polar chains that not only exhibit a stable Col phase but also an interesting two dimensional organization in graphite interfaces. ${ }^{24}$ In their system, the strong intermolecular interactions between intraannular polar groups prevent the molecules from sliding relative to each other thus stabilizing the Col phase, in the temperature range between $93-195^{\circ} \mathrm{C}$. This organic macromolecule is shown in Fig. 1 (a). A further system which exhibits a stable Col phase with hexagonal symmetry in the arrangement of the columns is the molecule reported by Shimura et al. (see Fig. 1 (b)). ${ }^{23}$ This is a SPM with intraannular oligooxyethylene chains and L-glutamic acid derivatives decorating the outer part of the ring. In this system the intraannular substituents are incompatible with the outer ring thus segregation between internal and external moieties is observed. In addition to this effect, the glutamic acid derivatives can produce intermolecular hydrogen bonds. Both effects, segregation and hydrogen bonding, apparently suppress the formation of the expected $\mathrm{N}$ phase to produce a stable Col phase, in the temperature range between $0-89{ }^{\circ} \mathrm{C}$ upon heating. Apart from the interaction between intraannular chains, the size of the SPMs also plays an important role and must be considered.

In both systems mentioned above, the two fundamental features required to stabilize the columnar LC phase are: the amphiphilic nature between the polar and non-polar groups promoting the segregation, and the reduction of the large internal void by the addition of polar groups in intraanular position. To be able to describe this complex behavior at a fundamental molecular level we have undertaken a systematic molecular-dynamics (MD) simulation study of a coarse-grained (CG) model that includes the effect of segregation (amphiphilic nature) between different parts of the SPM. This work is an extension of our previous work of CG model for undecorated SPMs,${ }^{26}$ where now intrannular grafted chains are considered. To describe a generic model of an amphiphilic SPM, the rigid macrocycles are built up by an hexagonal arrangement of softrepulsive spheres and the intraannular substituents are modeled as self-attracting Lennard-Jones chains in order to model the strongly attractive polar groups presented in real molecules. To the best of our knowledge, there are no reports in the literature of SPMs with extraannular polar groups showing LC behaviour. Nonetheless, we also present in this work the extrannular case in order to have a complete picture of the possible effect of the relative position of the polar groups attached to the ring.

The structure of the rest of the paper is organized as follows. In section II, both the intraannular and extraannular CG models for SPM are introduced together with the MD simulation details. In section III the order parameters used to identify LC phases and phase transitions are described. In section IV the MD configurations obtained upon cooling from high temperatures isotropic (I) states are discussed, and, finally, in section $\mathrm{V}$ a general discussion of this work is presented.

\section{Molecular models and simulation details}

The two models analyzed in this work are shown schematically in Fig. 1 (c) and Fig. 1 (d). Particles are composed of a rigid softrepulsive macrocycle grafted with flexible attractive chains. For both models, the rigid macrocycle is represented as an hexagonal arrangement of 24 beads of diameter $\sigma$ and mass $m$ while each of the 4 chains attached to structure are each modeled by 6 beads, of the same size and mass as the rigid macrocycle beads. Although the two models are composed of the same number of beads and similar interactions, they differ in the position where the chains are attached to the ring, i.e. they are isomer structures. The junctions between the first bead in each chain and the rigid macrocycles are rigid thus preventing the flipping of the chains with respect to the rigid structure. For example, in the model depicted in Fig. 1 (d) the chains are pointing outward with respect to the center of the ring and due to the rigid bonds between the chain and the macrocycles the chain itself can not fold back into the cavity of the molecule. Similarly, in the model 
shown in Fig. 1 (c) the chains are pointing inward without possibility for outward rotation.

The interactions between different beads are given in terms of a truncated and shifted Lennard-Jones (LJ) potential defined as:

$$
u(r)=\left\{\begin{array}{cc}
u_{L J}(r)-u_{L J}\left(r_{c}\right), & r \leq r_{c} \\
0, & r>r_{c}
\end{array}\right.
$$

with

$$
u_{L J}(r)=4 \varepsilon\left[\left(\frac{\sigma}{r}\right)^{12}-\left(\frac{\sigma}{r}\right)^{6}\right],
$$

where $r$ is the center-to-center distance between different beads, $\varepsilon$ is the well depth of the potential, and $r_{c}$ is a cut-off distance. Beads belonging to the rigid macrocycles interact via the aforementioned potential with $r_{c}=2^{1 / 6} \sigma$. This soft repulsive interaction coincides with the WCA reference potential. ${ }^{27}$ Rigid bonds are used between these beads in order to preserve the planar hexagonal structure. Interactions between the attached chains are given by eqn (1)-(2) with $r_{c}=2.5 \sigma$. The interaction between unlike beads is taken to be the same as the rigid backbone beadbead interaction, i.e. a soft-repulsive interaction, which gives rise to an amphiphilic character to the overall molecule. Beads along a chain are linked via a finitely extensible nonlinear elastic (FENE) springs given by: ${ }^{28,29}$

$$
u_{S}(r)=\frac{1}{2} k R_{0}^{2} \ln \left[1-\left(\frac{r}{R_{0}}\right)^{2}\right],
$$

where $k=30 \varepsilon / \sigma^{2}$ is the spring constant and $R_{0}=1.5 \sigma$ is the maximal bond length between bonded beads. This intramolecular potential is suitable to prevent the crossing between different chains and it is commonly used in molecular simulations of polymers using CG models.

Conventional reduced units are introduced in order to describe different thermodynamic states and structural properties. Reduced temperature, number density of beads, pressure and time are defined with respect to the $\mathrm{LJ}$ parameters as $T^{*}=k_{B} T / \varepsilon$, $\rho^{*}=N_{b} \sigma^{3} / V, P^{*}=P \sigma^{3} / \varepsilon$, and $t^{*}=t\left(\varepsilon / m \sigma^{2}\right)^{1 / 2}$, respectively, where $T$ is the absolute temperature, $k_{B}$ is the Boltzmann constant, $N_{b}$ is the total number of beads, $V$ is the system volume, $P$ is the pressure, and $t$ is the time. Similarly, all distances are given in units of $\sigma$. The model has a unique energy scale, $\varepsilon$, which defines the link between the experimental and simulated temperature scales. Whilst one could attempt to match this temperature to an observed quantity, e.g. a phase transition temperature, we have chosen otherwise. In this work we minimize the energy scales to reduce the computational effort and thus explore much longer time scales. The important energetic interactions are those between the side chains, which may be of the order of magnitude of hydrogen bonding association. The $\pi-\pi$ and dispersion interactions among the rings and the cross interactions are, in comparison, irrelevant in magnitude. ${ }^{30}$ This "scale-down" approach has been successfully applied in our group to study other polyphilic LC molecules ${ }^{31}$ and mixtures. ${ }^{32}$

Molecular Dynamics (MD) simulations of $N_{m}=496$ molecules (corresponding to $N_{b}=23808$ beads) were carried out in the N-P$\mathrm{T}$ ensemble employing a Nosé-Hoover thermostat ${ }^{33}$ and a Hoover barostat, using the Melchionna modification, ${ }^{34}$ in a cubic simulation box of volume $V$ with periodic boundary conditions.
All bonds in the rigid structure were kept constant using the SHAKE algorithm. ${ }^{35}$ Since cubic simulation box can prompt artificial LC structures, special care is taken when the off-diagonal elements of the pressure tensor are not fluctuating about zero. In this case the particular phase suffers stress that is removed by extending the simulations runs using the constant stress $\mathrm{N}-\sigma-\mathrm{T}$ ensemble, where fluctuations in the volume and in the simulation box shape are allowed. ${ }^{36}$ For all the simulations the leapfrog integration algorithm is used with a time step of $\delta t^{*} \approx 0.004$. The simulations are performed at a constant pressure of $P^{*}=0.10$ and a series of different states are run starting from low-density isotropic states at high temperature into highdensity states by cooling the system in a sequential manner. ${ }^{37,38}$ $1.0 \times 10^{6}$ time steps are used for equilibration and an additional $2.0 \times 10^{6}$ time steps are used to obtain averaged values. Near to the phase transition, more time steps are required in order to obtain reliable results. All MD simulations are performed using DL_POLY software. ${ }^{39,40}$

Phase transitions points observed by cooling of an isotropic fluid may in principle differ from those observed by the heating of pre-assembled structures. This hysteresis is expected in finite size simulations and is an enhanced manifestation of the metastability encountered experimentally in condensed phases. Thus, phase transition temperatures are hereby only roughly bracketed and no attempt has been made to locate them unequivocally, as that would require free energy calculations, ${ }^{41-43}$ or much larger system sizes to explore phase coexistence.

\section{Order parameters}

In order to detect possible formation of mesophases, different order parameters are used. The orientational order can be analyzed from the second-rank Saupe order tensor $\mathbf{Q}$, defined as: ${ }^{44-46}$

$$
\mathbf{Q}=\frac{1}{N_{m}} \sum_{j=1}^{N_{m}}\left(\frac{3}{2} \hat{\mathbf{u}}_{j} \otimes \hat{\mathbf{u}}_{j}-\frac{\mathbf{I}}{2}\right),
$$

where $\mathbf{u}_{j}$ is the principal molecular axis (which is perpendicular to the ring, i.e., is oriented out of the plane of the page in Fig. 1) of molecule $j, \otimes$ denotes dyadic product, and $\mathbf{I}$ is the second-rank unit tensor. Diagonalization of the tensor yields the eigenvalues $\lambda^{+} \geq \lambda^{0} \geq \lambda^{-}$and the corresponding orthonormal eigenvectors $\hat{\mathbf{n}}^{+}$, $\hat{\mathbf{n}}^{\mathbf{0}}$, and $\hat{\mathbf{n}}^{-}$. The nematic order parameter, $S$, is defined as the dominant eigenvalue (i.e., the eigenvalue of greatest absolute value) of $\mathbf{Q}$ and the corresponding eigenvector is the preferred direction for the system, which is called the system director. ${ }^{46}$ Depending on the sign of $S$, two different scenarios in the order of the system can be observed. ${ }^{47,48}$ First, if $0<S \leq 1$, the particles are preferentially aligned along the principal director, corresponding to conventional $\mathrm{N}$ order. On the other hand, if $-0.5 \leq$ $S<0$, the particles are preferentially aligned in a plane perpendicular to the principal director. The latter case is sometimes called antinematic order. The formation of a possible second preferential direction in the system (biaxial order) can be identified using the difference $\Delta$ between the remaining two smallest eigenvalues of the matrix $\mathbf{Q}$. If they are degenerate, all directions perpendicular to the director are equally probable and the system has unaxial symmetry $(\Delta \sim 0)$. 
The possible formation of layers in the system was monitored using the translational order parameter $\tau$, obtained from simulation by means of the following expression:

$$
\tau(\boldsymbol{k})=\left\langle\left|\frac{1}{N_{m}} \sum_{j=1}^{N_{m}} \exp \left(\imath \boldsymbol{k} \cdot \boldsymbol{r}_{j}\right)\right|\right\rangle,
$$

where $\mathbf{r}_{j}$ is the position of the rigid backbone center of mass of molecule $j, \mathbf{k}=2 \pi \hat{\mathbf{l}} / d_{l}$ is the reciprocal lattice vector, $\hat{\text { lis }}$ the unit vector normal to the plane formed by the layers, and $d_{l}$ is the smectic layer spacing. The parameter $\tau$ corresponds to the amplitude of the smectic layers and can be identified as the first coefficient in the Fourier expansion of the particle density. ${ }^{49,50}$ The layer direction $\hat{\mathbf{l}}$ can be obtained from the eigenvectors of the matrix $\mathbf{Q}$ and the periodicity of the layers $d_{l}$ can be calculated evaluating eqn (5) for different trial layer spacing in a convenient range, taking $d_{l}$ as the value that maximizes the expression. ${ }^{50,51}$

In order to detect the formation of $\mathrm{Col}$ phases, in this case with hexagonal bidimensional arrangement, the nematic order parameter, $S$, together with the hexagonal bond-orientational order parameter, $\psi_{6}$, may be used. The latter is defined as: $:^{52,53}$

$$
\psi_{6}=\left\langle\left|\frac{1}{N_{m}} \sum_{i=1}^{N_{m}} \frac{1}{N_{i}} \sum_{j} \exp \left(\imath 6 \theta_{i j}\right)\right|\right\rangle
$$

where the sum on $j$ is over the $N_{i}$ nearest neighbors of particle $i$ and $\theta_{i j}$ is the angle between the projection of the vector $\mathbf{r}_{i j}$ joining the rigid backbone center of mass of the $i$ th and $j$ th molecules in the plane perpendicular to the director and an arbitrary but fixed axis in the same plane. This parameter can take the values in the range $0 \leq \psi_{6} \leq 1, \psi_{6}=1$ being the case in which the system has a perfect hexagonal order and $\psi_{6}=0$ in the absence of such an order.

Finally, to support the information obtained by the previous order parameters, two different pair correlation functions have been calculated to aid the observation of the phase transitions. ${ }^{7,54,55}$ In order to detect the formation of smectic phases, the projection of the molecular pair distribution function along the system director, $g_{\|}\left(r_{\|}\right)$is evaluated. This correlation function gives information about the probability of finding a molecule in cylindrical shells along the direction of the layers. Similarly for the formation of columns, the columnar pair correlation function, $g_{c}\left(r_{\|}\right)$, provides information about the probability of finding a molecule in a slab of a cylinder along the director. This function allows the detection of longitudinal density correlations typically observed in Col phases. $g_{\|}\left(r_{\|}\right)$and $g_{c}\left(r_{\|}\right)$have similar structure, however, the latter is restricted only to particles lying in cylindrical shells of diameter similar to the diameter of the columns. In both functions, $r_{\|}$is defined as the projection of distance between the centers of mass of two rings along the system director.

\section{Results}

\section{A. Extraannular SPMs}

In Fig. 2 results for the number density, $\rho^{*}$, the nematic order parameter, $S$, the translational order parameter, $\tau$, and biaxial order parameter, $\Delta$, as a function of the temperature, $T^{*}$, are shown for the model of SPM with extraannular flexible chains.

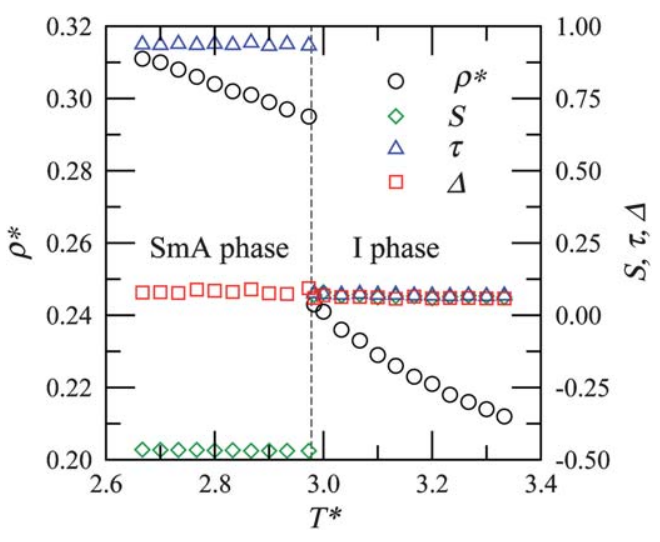

Fig. 2 Number density, $\rho^{*}$, nematic order parameter, $S$, translational order parameter, $\tau$, and biaxial order parameter, $\Delta$, as a function of the temperature $T^{*}$, for a system of SPMs with extraannular chains at a pressure $P^{*}=0.100$. The dashed line is used to delineate the I and SmA domains. Data was obtained cooling from a high temperature I state to a SmA state. Error bars are smaller than the symbols.

Upon cooling from an initial low density state, this system exhibits a first order phase transition from an isotropic (I) state $\left(\rho^{*}=0.243 \pm 0.003, S=0.070 \pm 0.026, \tau=0.076 \pm 0.024\right)$ to a smectic-A $(\mathrm{SmA})$ state $\left(\rho^{*}=0.295 \pm 0.002, S=-0.469 \pm\right.$ $0.003, \tau=0.933 \pm 0.024)$ at temperature $T^{*} \sim 2.978$. This transition was verified by discontinuities in the density, and in the nematic and translational order parameters. The I-SmA phase transition for this system can also be monitored with the aid of the pair correlation function along the system director, $g_{\|}\left(r_{\|}\right)$, which is shown in Fig. 3. In this figure the SmA phase is characterized by the formation of peaks corresponding to density correlations along the direction of the layers. The distance between neighbor peaks provides the interlayer separation, in this case corresponds approximately to $d_{l} \approx 10 \sigma$, which coincides with the distance obtained by means of eqn (5).

In Fig. 4 configurations for the highest-density I state and lowest-density SmA state are depicted, where the formation of layers at low temperatures is apparent. This SmA phase is similar to the phase found in our previous work of ungrafted SPM, ${ }^{26}$ where the orientations of the principal molecular axis of the rings

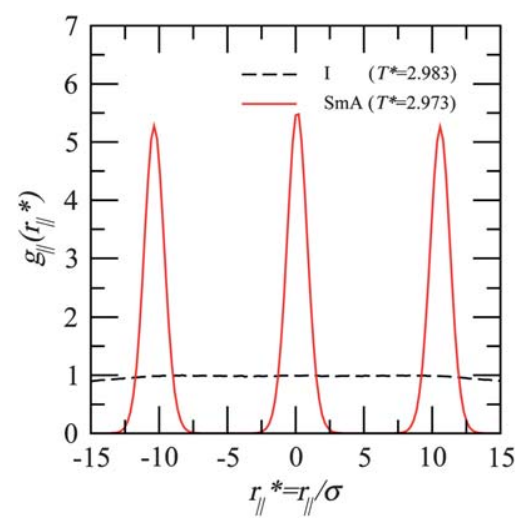

Fig. 3 Projection of the pair correlation function along the system director for a system of SPMs with extraannular chains at a pressure $P^{*}=$ 0.100 . Dashed line corresponds to the highest-density I state at temperature $T^{*}=2.983$, and continuous line corresponds to the lowest-density SmA state at temperature $T^{*}=2.983$. 


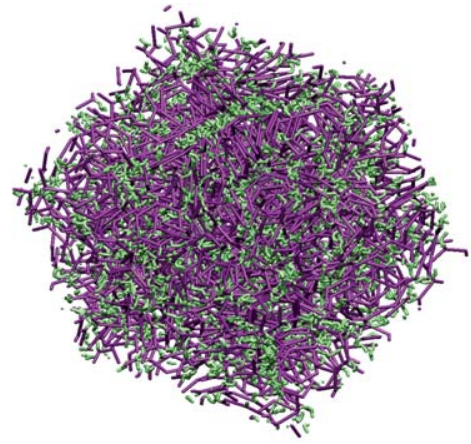

(a)

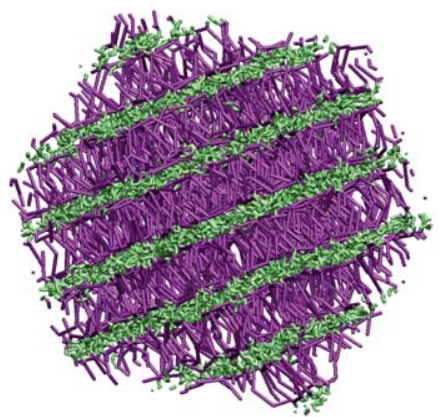

(b)

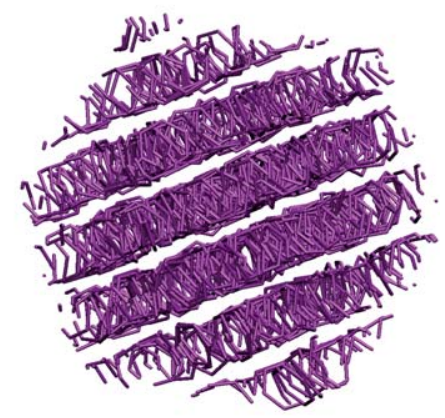

(c)

Fig. 4 Snapshots for two different configurations for SPMs with extraannular chains at a pressure $P^{*}=0.1$. (a) Highest-density I state at $T^{*}=2.983$; (b) lowest density SmA state at $T^{*}=2.973$; (c) the same state as (b) omitting the flexible chains to aid visualization of the layers. In the three snapshots, thick lines replace cores of the SPMs. Color scheme as in Fig. 1.

are mainly perpendicular to the system director (perpendicular to the layers) with negative values of the nematic order parameter, $S$. LC states with negative values of the nematic order parameter are sometimes called antinematic phases. ${ }^{47,48}$ The absence of order amongst the rings in each layer is evident by the low values of the biaxial order parameter, $\Delta$ (see Fig. 2), confirming that the system preserves cylindrical symmetry typical of SmA phases. This phase transition is presumably driven by two factors. The first one is the segregation between the rigid soft-repulsive spheres and the attractive extraannular chains, in form of layers, similar to the segregation observed in block copolymers models. ${ }^{56}$ The second effect is due to the frustration between the molecule anisotropy (non-convex shape) and the empty space inside the ring that prevents the alignment of the molecules, i.e., particles can partially occupy some of the free volume within neighbor annular regions suppressing the formation of $\mathrm{N}$ or $\mathrm{Col}$ phases. ${ }^{24,26}$ In systems without grafted chains, this antinematic behavior is seen only if the ring cavity is of such large dimensions that the rings can interpenetrate if their molecular axes are not aligned, i.e. for smaller cavities or thicker donut-like geometries, only columnar and/or nematic aligned phases are possible. ${ }^{26}$

The mobility of the SmA phase was examined by monitoring the mean-square displacement (MSD) of the center of mass of particles, and the analog $\mathrm{MSD}_{\perp}$ and $\mathrm{MSD}_{\|}$which are the meansquare displacements in directions perpendicular and parallel to the system director, respectively. ${ }^{57}$ In Fig. 5 (a) these quantities are shown for the lowest-density SmA state where it can be observed that the system presents high diffusion of particles in the plane of the layers (perpendicular to the director). The diffusion of rings between neighbor layers (parallel to the director) seems to be restricted by the strong interactions between segregated chains at low temperatures as can be observed in the behavior of $\mathrm{MSD}_{\|}$in the same figure.

\section{B. Intraannular SPMs}

The model of SPM with intraannular flexible chains, presents a contrasting behavior with respect to the mesophases observed. In Fig. 6 results for the number density, $\rho^{*}$, the nematic order parameter, $S$, and the bond-orientation order parameter, $\psi_{6}$, as

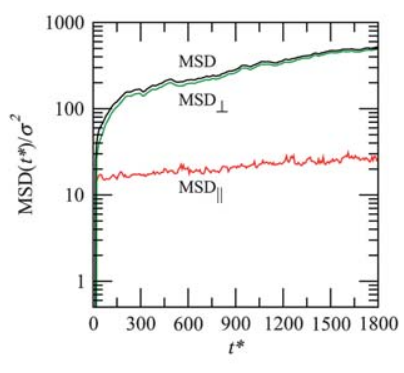

(a)

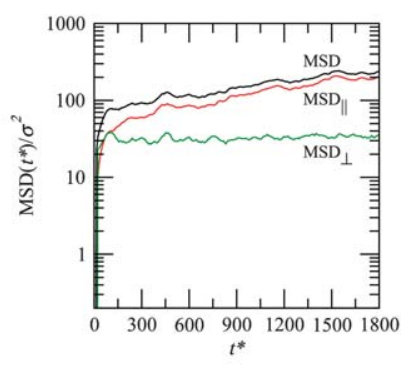

(b)
Fig. 5 Mean square displacements $\mathrm{MSD}, \mathrm{MSD}_{\perp}$ and $\mathrm{MSD}_{\|}$, for the system of SPMs at a pressure $P^{*}=0.1$. (a) Lowest-density SmA state at temperature $T^{*}=2.973$ for SPMs with extraannular chains; (b) lowestdensity $\mathrm{Col}_{h}$ state at temperature $T^{*}=2.517$ for SPMs with intraannular chains.

a function the temperature, $T^{*}$, are shown for this model. Upon cooling, the system exhibits a first order phase transition from an I state $\left(\rho^{*}=0.390 \pm 0.006, S=0.169 \pm 0.046, \psi_{6}=0.082 \pm\right.$ $0.041)$ to a hexagonal-disordered columnar $\left(\mathrm{Col}_{\text {hd }}\right)$ state $\left(\rho^{*}=\right.$ $\left.0.471 \pm 0.006, S=0.919 \pm 0.010, \psi_{6}=0.871 \pm 0.013\right)$ at temperature $T^{*} \sim 2.525$. At low temperatures, the high and positive values of the nematic order parameter, $S$, evidence the alignment of the system in a preferential direction while high values of the bond-orientational order parameters, $\psi_{6}$, show that the system starts to order with hexagonal symmetry in the plane perpendicular to the system director.

Further information about the structure of the Col phase can be inferred analyzing the columnar pair correlation function along the system director, $g_{c}\left(r_{\|}\right)$, which is shown in Fig. 7. In this figure the formation of peaks due to the density-correlations along the columns is perceptible, however these correlations are lost at distances along the director larger than about $r_{\|}^{*} \sim 8$. This liquid-like structure observed within the columns is in fact a characteristic of a $\mathrm{Col}_{\text {hd }}$ phase. ${ }^{58}$ Further cooling of this system preserves the $\mathrm{Col}_{\text {hd }}$ phase as can be seen in Fig. 7, where $g_{c}\left(r_{\|}\right)$for the highest-density $\mathrm{Col}_{\text {hd }}$ state is shown. As expected, at low temperatures the $\mathrm{Col}_{\text {hd }}$ phase compacts and the density correlations along the columns are enhanced. 


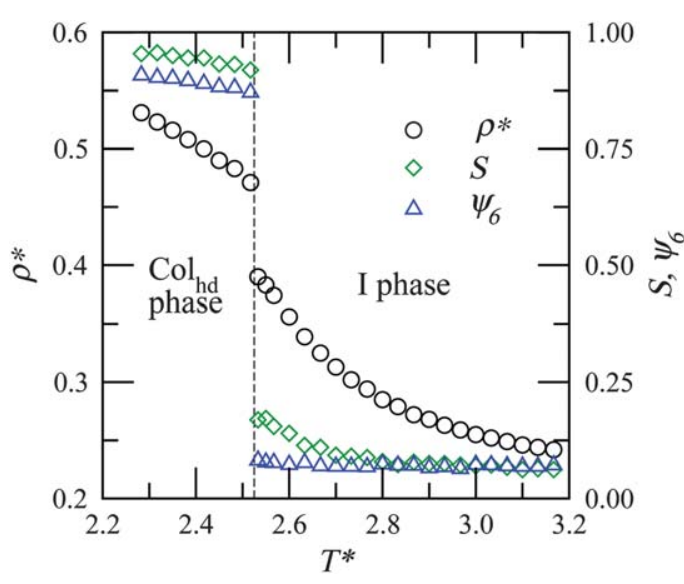

Fig. 6 Number density, $\rho^{*}$, nematic order parameter, $S$, and bondorientational order parameter, $\psi_{6}$, as a function of the temperature $T^{*}$, for a system of SPMs with intraannular chains at a pressure $P^{*}=0.100$. The dashed line is used to delineate the $\mathrm{I}$ and $\mathrm{Col}_{\mathrm{hd}}$ domains. Data was obtained by cooling from a high temperature I state to a $\mathrm{Col}_{\mathrm{hd}}$ state. Error bars are smaller than the symbols.

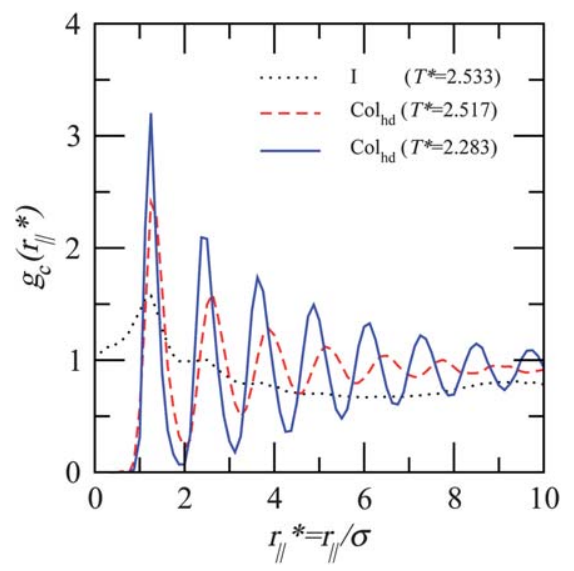

Fig. 7 Columnar pair correlation function along the system director for a system of SPMs with intraannular chains at a pressure $P^{*}=0.100$. Dot line corresponds to the highest-density I state at temperature $T^{*}=2.533$, dashed line corresponds to the lowest-density $\mathrm{Col}_{\text {hd }}$ state at temperature $T^{*}=2.517$ and continuous line corresponds to the highest-density $\mathrm{Col}_{\mathrm{hd}}$ state at temperature $T^{*}=2.283$.

Additional comments about the MD simulation details in the vicinity of the $\mathrm{I}-\mathrm{Col}_{\mathrm{hd}}$ should be mentioned. First, when the system is cooling down from the highest-density I state $\left(T^{*}=\right.$ 2.533 ) to the $\mathrm{Col}_{\text {hd }}$ state ( $\left.T^{*}=2.517\right)$, MD molecular simulations in the N-P-T ensemble were used. However, the Col phase formed presents some degree of stress where the particles appeared to be tilted with respect to the direction of the columns. Restarting the simulation in the $\mathrm{N}-\sigma-\mathrm{T}$ ensemble proved the metastability of the aforementioned structures. In the final stable state the columns of the system tend to align along the system director for the non-cubical simulation box generated by this ensemble, and, in addition, the density is also increased considerably compared with the standard N-P-T simulations.

In Fig. 8 snapshots for two different states corresponding to an I state and $\mathrm{a} \mathrm{Col}_{\text {hd }}$ are shown, with tubular structures evident at low temperatures. The mobility in this system was followed through the MSD and their projections along and perpendicular to the system director, which are depicted in Fig. 5 (b). The MSD reveals that the diffusion of the particles along the columns is high, while the diffusion in the direction perpendicular to the columns is slower, as expected for $\mathrm{Col}_{\text {hd }}$ phases where the system behaves as a one-dimensional liquid in the direction of the columns and as a solid with 2D six-fold orientation order in the perpendicular direction. In other words, as the structure is densely packed, this result presumably points to the sliding of the columns with respect to each other and the persistence of the columnar structure. The overall behavior in SPMs with intraannular groups reported in this work is comparable to the experimental observations of Fischer et al. ${ }^{24}$ and Shimura et al.,${ }^{23}$ where the effect of chain segregation promotes the formation of a stable Col phase.

\section{Discussion}

We have reported MD simulations results for two different generic CG models of amphiphilic SPMs with intraannular and extrannular self-attracting grafted chains, respectively. It has been found that the supramolecular organization of the particles depends strongly on the position of the chains attached to the rigid ring structure. Both models attempt to describe the basic salient features of recent reported examples of SPMs with polar attractive chains. ${ }^{23,24}$ In our CG models the relatively weak $\pi-\pi$ interactions between aromatic cores belonging to the ring have been smeared out in the form of soft-repulsion potential and the polar attractions between chains have been taken as a LJ potential, in accordance with the amphiphilic nature of the target molecules.

Upon quenching from an I state, SPMs with extraannular chains exhibit a first order I-SmA phase transition in which the segregation of the chains induce the formation of a unique lamellar structure. The $\mathrm{SmA}$ structure resembles what is observed for ungrafted SPMs, ${ }^{26}$ where particles spontaneously tend to align perpendicularly to the direction of the layers showing antinematic order. We are not aware of any experimental system in the literature that allows for a qualitative comparison. A related molecule with similar topology is the system reported by Höger et al., ${ }^{15}$ however the molecules described on that work are SPM with alkane side chains, i.e. they do not present polar extraannular groups. Höger et al. do not observe the formation of any columnar or nematic LC phases, something that is in agreement with our simulations. Notwithstanding, the related SmA LC phase seen in simulation has not been observed experimentally presumably because the alkyl chains that they reported do not self-attract strongly enough to observe the segregation into a lamellar phase.

On the other hand, our CG model of amphiphilic SPM with intraannular chains, upon quenching, reveals the presence of an I$\mathrm{Col}_{\text {hd }}$ phase transition. The formation of columns, rather than mere alignment in $\mathrm{N}$ order, is favoured by means of two factors: the reduction of the macrocycle cavity by the intraannular substituents and the self-attraction of inner parts of the molecules preventing the molecules from sliding relative to each other. Experimentally, the same behavior has been reported in SPM with intraannular polar chains by Fischer et al., ${ }^{24}$ and by Shimura et al., ${ }^{23}$ where the same segregation effect promotes the 


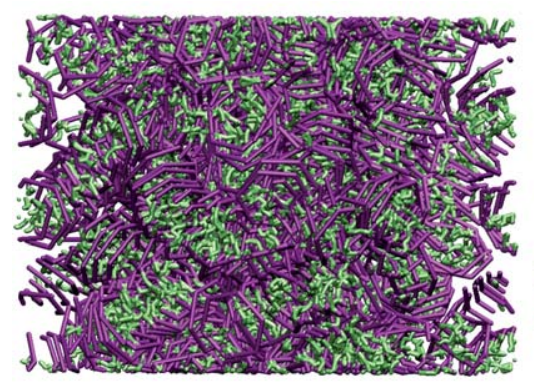

(a)

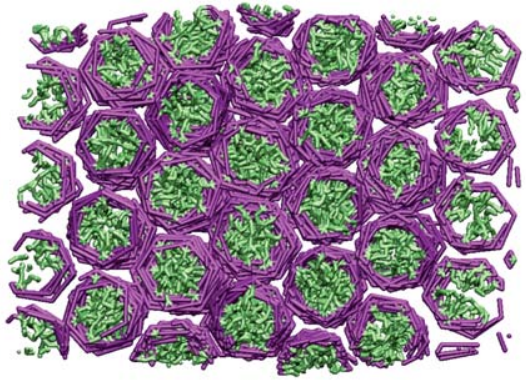

(b)

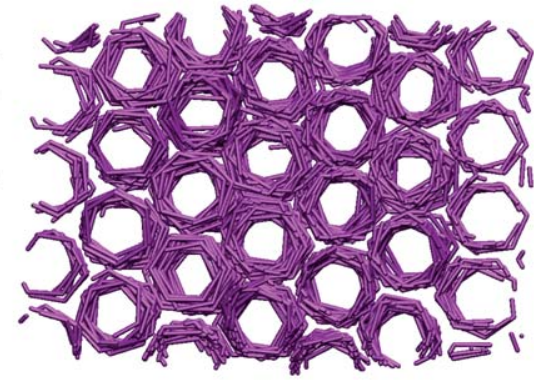

(c)

Fig. 8 Snapshots for two different configurations for SPMs with intraannular chains at a pressure $P^{*}=0.1$. (a) Highest-density I state at $T^{*}=2.533$; (b) lowest-density $\mathrm{Col}_{\text {hd }}$ state at $T^{*}=2.517$; (c) the same state as (b) omitting the flexible chains to aid visualization of the tubular structures. Snapshots are drawn in the same fashion as in Fig. 4.

stabilization of the $\mathrm{Col}_{\text {hd }}$ phase. This qualitative agreement between experiments and simulations results validates, in some way, how our relatively simple molecular model can be used to study the most important features on the self-assembly of SPMs. We have attempted to simulate the intermediate system, in which the grafted chains are not restricted to be inside or outside the rigid backbone. This freely rotating system does not present any LC behaviour at the thermodynamic conditions we have explored. However, more systematic study is required in that direction to fully understand its behavior. Moreover, questions are still open with respect to the impact of the supramolecular arrangement of SPM with different functional groups in both the rigid structure and the grafted groups attached to the rings and to the effect of solvents on the self-assembly. Future work will address these issues.

\section{Acknowledgements}

Stimulating discussions with Prof. Dr. Sigurd Höger (University of Bonn) are gratefully acknowledged. Financial support for this work has been given by the U.K. Engineering and Physical Sciences Research Council (EPSRC), grant EP/E016340, "Molecular System Engineering". Simulations were performed using the facilities of the Imperial College High Performance Computing Service.

\section{References}

1 S. Chandrasekhar, B. K. Sadashiva and K. A. Suresh, Pramana, 1977, 9, 471.

2 R. J. Bushby and O. R. Lozman, Curr. Opin. Colloid Interface Sci., $2002,7,343$.

3 S. Kumar, Chem. Soc. Rev., 2006, 35, 83.

4 L. Onsager, Ann. N. Y. Acad. Sci., 1949, 51, 627.

5 G. J. Vroege and H. N. W. Lekkerkerker, Rep. Prog. Phys., 1992, 55, 1241.

6 D. Frenkel and R. Eppenga, Phys. Rev. Lett., 1982, 49, 1089.

7 J. A. C. Veerman and D. Frenkel, Phys. Rev. A: At., Mol., Opt. Phys., 1992, 45, 5632.

8 M. A. Bates, J. Chem. Phys., 1999, 111, 1732.

9 F. M. van der Kooij and H. N. W. Lekkerkerker, J. Phys. Chem. B, $1998, \mathbf{1 0 2}, 7829$.

10 F. M. van der Kooij, K. Kassapidou and H. N. W. Lekkerkerker, Nature, 2000, 406, 868.

11 F. M. van der Kooij and H. N. W. Lekkerkerker, Philos. Trans. $R$. Soc. London, Ser. A, 2001, 359, 985.
12 W. K. Kegel and A. van Blaaderen, Science, 2000, 287, 290.

13 J. S. Moore, Acc. Chem. Res., 1997, 30, 402.

14 S. Höger, J. Polym. Sci., Part A: Polym. Chem., 1999, 37, 2685.

15 S. Höger, V. Enkelmann, K. Bonrad and C. Tschierske, Angew. Chem., Int. Ed., 2000, 39, 2267.

16 C. Tschierske, Annu. Rep. Prog. Chem., Sect. C, 2001, 97, 191.

17 C. Grave and A. D. Schluter, Eur. J. Org. Chem., 2002, 3075.

18 D. H. Zhao and J. S. Moore, Chem. Commun., 2003, 807.

19 S. Rosselli, A. D. Ramminger, T. Wagner, G. Lieser and S. Hoger, Chem.-Eur. J., 2003, 9, 3481.

20 S. Höger, Chem.-Eur. J., 2004, 10, 1320.

21 S. Höger, X. H. Cheng, A. D. Ramminger, V. Enkelmann, A. Rapp, M. Mondeshki and I. Schnell, Angew. Chem., Int. Ed., 2005, 44, 2801.

22 W. Zhang and J. S. Moore, Angew. Chem., Int. Ed., 2006, 45, 4416.

23 H. Shimura, M. Yoshio and T. Kato, Org. Biomol. Chem., 2009, 7, 3205.

24 M. Fischer, G. Lieser, A. Rapp, I. Schnell, W. Mamdouh, S. De Feyter, F. C. De Schryver and S. Höger, J. Am. Chem. Soc., 2004, 126, 214

25 S. Höger, J. Weber, A. Leppert and V. Enkelmann, Beilstein J. Org. Chem., 2008, 4, DOI: 10.1186/1860-5397-4-1.

26 C. Avendaño and E. A. Müller, Phys. Rev. E: Stat., Nonlinear, Soft Matter Phys., 2009, 80, 061702.

27 J. D. Weeks, D. Chandler and H. C. Andersen, J. Chem. Phys., 1971, 54, 5237.

28 G. S. Grest and K. Kremer, Phys. Rev. A: At., Mol., Opt. Phys., 1986, 33, 3628 .

29 L. Lenoci and P. J. Camp, J. Am. Chem. Soc., 2006, 128, 10111.

30 E. A. Müller and K. E. Gubbins, Ind. Eng. Chem. Res., 2001, 40, 2193.

31 A. J. Crane, F. J. Martínez-Veracoechea, F. A. Escobedo and E. A. Müller, Soft Matter, 2008, 4, 1820.

32 A. J. Crane and E. A. Müller, Faraday Discuss., 2010, 144, 187.

33 W. G. Hoover, Phys. Rev. A: At., Mol., Opt. Phys., 1985, 31, 1695.

34 S. Melchionna, G. Ciccotti and B. L. Holian, Mol. Phys., 1993, 78, 533.

35 J. P. Ryckaert, G. Ciccotti and H. J. C. Berendsen, J. Comput. Phys., 1977, 23, 327.

36 M. Parrinello and A. Rahman, Phys. Rev. Lett., 1980, 45, 1196.

37 L. D. Gelb and E. A. Müller, Fluid Phase Equilib., 2002, 203, 1.

38 F. Martínez-Veracoechea and E. A. Müller, Mol. Simul., 2005, 31, 33.

39 W. Smith, Mol. Simul., 2006, 32, 933.

40 W. Smith, I. T. Forester, and T. R. Todorov, The DL_POLY_2 user manual, CCLRC, Daresbury Laboratory, Daresbury, Warrington, U.K. (2009).

41 P. Bolhuis and D. Frenkel, J. Chem. Phys., 1997, 106, 666.

42 M. Müller and K. C. Daoulas, J. Chem. Phys., 2008, 128, 024903.

43 C. Vega, E. Sanz, J. L. F. Abascal and E. G. Noya, J. Phys.: Condens. Matter, 2008, 20, 153101.

44 A. Saupe, Angew. Chem., Int. Ed. Engl., 1968, 7, 97.

45 M. P. Allen, G. T. Evans, D. Frenkel and B. M. Mulder, Adv. Chem. Phys., 1993, 86, 1. 
46 R. J. Low, Eur. J. Phys., 2002, 23, 111.

47 H. H. Wensink and G. J. Vroege, Phys. Rev. E: Stat, Nonlinear, Soft Matter Phys., 2005, 72, 031708.

48 D. van der Beek, P. Davidson, H. H. Wensink, G. J. Vroege and H. N. W. Lekkerkerker, Phys. Rev. E: Stat., Nonlinear, Soft Matter Phys., 2008, 77, 031708.

49 S. Singh, Phys. Rep., 2000, 324, 107.

50 R. E. Webster, N. J. Mottram and D. J. Cleaver, Phys. Rev. E: Stat., Nonlinear, Soft Matter Phys., 2003, 68, 021706.

51 M. A. Bates and G. R. Luckhurst, J. Chem. Phys., 1999, 110, 7087.
52 P. M. Chaikin and T. C. Lubensky, Principles of Condensed Matter Physics (Cambridge University Press, England, 1995).

53 C. Das, A. K. Sood and H. R. Krishnamurthy, Phys. A, 1999, 270, 237.

54 H. Zewdie, Phys. Rev. E: Stat. Phys., Plasmas, Fluids, Relat. Interdiscip. Top., 1998, 57, 1793.

55 P. L. Cristinziano and F. Lelj, J. Chem. Phys., 2007, 127, 134506.

56 K. Binder and M. Müller, Curr. Opin. Colloid Interface Sci., 2000, 5, 314.

57 M. A. Bates and G. R. Luckhurst, J. Chem. Phys., 2004, 120, 394.

58 P. T. Collings and M. Hird, Introduction to Liquid Crystals (Taylor \& Francis, London, 1997). 